\title{
Comparative Evaluation of Effectiveness of IAVchip DNA Microarray in Influenza A Diagnosis
}

\author{
K. T. Sultankulova, ${ }^{1}$ O. V. Chervyakova, ${ }^{1}$ N. S. Kozhabergenov, ${ }^{1}$ K. A. Shorayeva, ${ }^{1}$ \\ V. M. Strochkov, ${ }^{1}$ M. B. Orynbayev, ${ }^{1}$ N. T. Sandybayev, ${ }^{1}$ A. R. Sansyzbay, ${ }^{1}$ and A. V. Vasin ${ }^{2}$ \\ ${ }^{1}$ Research Institute for Biological Safety Problems (RIBSP), Science Committee of Ministry of Education and Science of \\ Republic of Kazakhstan, Gvardeiskiy, Kordaiskiy Rayon, Zhambylskaya Oblast 080409, Kazakhstan \\ ${ }^{2}$ Research Institute of Influenza, Ministry of Healthcare of the Russian Federation, Prof. Popov Street 15/17, \\ Saint Petersburg 197376, Russia
}

Correspondence should be addressed to K. T. Sultankulova; sultankul70@mail.ru

Received 4 July 2014; Revised 22 September 2014; Accepted 28 September 2014; Published 23 November 2014

Academic Editor: Peirong Jiao

Copyright (C) 2014 K. T. Sultankulova et al. This is an open access article distributed under the Creative Commons Attribution License, which permits unrestricted use, distribution, and reproduction in any medium, provided the original work is properly cited.

\begin{abstract}
The paper describes comparative evaluation of IAVchip DNA microarray, reverse transcription PCR (RT-PCR), and real-time RTPCR versus virus isolation in chicken embryos and shows their diagnostic effectiveness in detection and subtyping of influenza A virus. The tests were evaluated with use of 185 specimens from humans, animals, and birds. IAVchip DNA microarray demonstrates higher diagnostic effectiveness (99.45\%) in early influenza A diagnosis as compared to the real-time PCR (98.38\%) and RT-PCR (96.22\%), thus showing its clear superiority. Diagnostic sensitivity of IAVchip DNA microarray (100\%) exceeds the same of RTPCR (95.95\%) and real-time RT-PCR (97.96\%) in the range of estimated confidence intervals. IAVchip DNA microarray and realtime RT-PCR displayed equal diagnostic specificity (98.85\%), while diagnostic specificity of RT-PCR was 96.40\%. IAVchip DNA microarray has an advantage over the other tests for influenza A diagnosis and virus identification as a more rapid method that allows performing simultaneous detection and subtyping of about tens of specimens within one experiment during 8-10 hours. The developed IAVchip DNA microarray is a general test tool that enables identifying simultaneously 16 hemagglutinin (HA) and 9 neuraminidase (NA) subtypes of influenza A virus and also to screen the influenza A viruses from humans, animals, and birds by $\mathrm{M}$ and NP genes.
\end{abstract}

\section{Background}

Influenza A virus (IAV) belongs to Orthomyxoviridae family. IAV is subtyped according to antigenic specificity of the surface glycoproteins hemagglutinin (HA) and neuraminidase (NA). Today 16 hemagglutinin and 9 neuraminidase subtypes are known [1]. Influenza A is contagious both for humans and animals. All IAV subtypes have been detected among water birds that are a natural IAV reservoir. The most frequent are 24 combinations of hemagglutinin and neuraminidase: H1N1, H2N2, H2N3, H3N2, H3N8, H4N2, H4N4, H4N6, H4N8, H5Nl, H5N2, H5N9, H6Nl, H6N2, H6N5, H6N9, H7N1, H7N2, H7N3, H7N7, H9N2, H9N8, H10N7, and H11N9. Among horses influenza is caused by viruses of subtypes $\mathrm{H} 7 \mathrm{~N} 7$ and $\mathrm{H} 3 \mathrm{~N} 8$. Equine influenza virus of $\mathrm{H} 3 \mathrm{~N} 8$ subtype causes disease in dogs $[2,3]$. Pigs are infected with influenza virus of subtypes H1N1, H1N2, H3N1, H3N2, and H2N3. The same viruses can affect humans. Only 3 HA subtypes $(\mathrm{H} 1, \mathrm{H} 2$, and $\mathrm{H} 3)$ and $2 \mathrm{NA}(\mathrm{N} 1, \mathrm{~N} 2)$ have been detected among humans during the last century [4]. However cases of infection among humans with highly pathogenic IAV of subtypes such as $\mathrm{H} 5 \mathrm{~N} 1, \mathrm{H} 7 \mathrm{~N} 7, \mathrm{H} 9 \mathrm{~N} 2$, and $\mathrm{H} 7 \mathrm{~N} 9$ are recorded recently [5-8].

Owing to the high mutation rate of the surface HA and NA glycoproteins of IAV subtyping appears to be difficult; therefore, it is necessary to use supplementary methods and tools of diagnosis as well as their combinations to get reliable results $[4,8]$. High pathogenicity of influenza viruses and great economic and social damage caused by the infection requires rapid and correct diagnosing. 
So far, the DNA microarray-based detection is one of promising techniques in differential diagnosis of IAV infection, since it allows combining efficiency of the nucleic acid amplification and ample capacities of screening with use of biochip [9-14].

\section{Objectives}

IAVchip DNA microarray $[15,16]$ for early IAV detection and differentiation has been developed at the RIBSP (Research Institute for Biological Safety Problems) and now the ways of its use in practice are investigated. The objective of the study was to evaluate diagnostic effectiveness of IAVchip DNA microarray in differential detection of 16 hemagglutinin and 9 neuraminidase IAV subtypes as compared to virus isolation in chicken embryos, real-time PCR, and RT-PCR.

\section{Study Design}

3.1. Tested Samples. Smears and swabs from humans, animals, and birds (185 samples in total) were used as a test subject. Forty-two human samples were taken in Kazakhstanean hospitals under the Program of Epidemiological Surveillance in 2012 from cases suspected for acute respiratory viral infection and were kindly provided to our laboratory by the Republican sanitary-epidemiological station.

Eighty-five samples were collected in 2012 from horses with signs of a respiratory infection in Kostanaiskaya, Almatinskaya, and Zhambylskaya oblasts under the Republican Science-and-Technology Program "Epizootiological monitoring of the territory of Kazakhstan, Central Asian and Neighboring Countries for Equine Influenza”.

Fifty-eight samples from wild birds were taken in 20052006 and in 2012 in Akmolinskaya and Karagandinskaya oblasts under the Program of Avian Influenza Surveillance.

Sampling was carried out following WHO recommendations [17]. Nasal smears (swabs) were taken from humans and horses using dry sterile probes with cotton tips; cloacal swabs were taken from birds in the same way. After sampling the cotton pellet (working part of the probe) was placed into a cryotube with sterile transport medium (transport media consisted of Hanks balanced salt solution supplemented with $10 \%$ glycerol, $200 \mathrm{U} / \mathrm{mL}$ penicillin, $200 \mathrm{mg} / \mathrm{mL}$ streptomycin, $100 \mathrm{U} / \mathrm{mL}$ polymyxin B sulphate, $250 \mathrm{mg} / \mathrm{mL}$ gentamicin, and $50 \mathrm{U} / \mathrm{mL}$ nystatin). Cryotubes with samples were transported in liquid nitrogen $\left(-196^{\circ} \mathrm{C}\right)$.

\subsection{Control Samples. One has the following:}

(i) plasmid DNA containing IAV M gene;

(ii) plasmid DNA containing IAV NP gene.

M gene (EU213070.2) and NP gene (EU213048.1) were cloned into strain XL1-Blue E. coli using pGEM-T VectorSystems (Promega). After that plasmid DNAs containing $M$ and NP genes of influenza A virus were extracted using QIAprep Spin Miniprep Kit (Qiagen).

\subsection{Assay of Samples with Use of IAVchip DNA Microarray for Influenza A Diagnosis and Virus Subtyping}

3.3.1. RNA Extraction. RNAs were extracted from samples with the help of TRizol ("Invitrogen," USA) following the manufacturer's instructions.

3.3.2. Viral RNA Amplification. One-step RT-PCR was performed with use of Super Script III Platinum One-Step Quantitative RT-PCR System (“Invitrogen," USA) following the manufacturer's instructions. The universal primer pair selected for the terminal highly conservative regions that are present in all IAV genome segments (MBTuni-12 and MBTuni-13) has been used in multisegment amplification [18]. Conditions of RT-PCR are as follows: SuperScriptIII RT/PlatinumR Taq Mix-1 $\mu \mathrm{L}$; 2X Reaction Mix-25 $\mu \mathrm{L}$; primers $10 \mu \mathrm{M}-1.4 \mu \mathrm{L}$ each; RNaseOUT $-1 \mu \mathrm{L}$; RNA (1 pg to $1 \mu \mathrm{g})-10 \mu \mathrm{L}$; DEPC-treated water up to $50 \mu \mathrm{L}$ [15]. Fluorescent labeling was made via direct integration of Cy5-dCTP ("DNA-Synthesis," Russia) immediately in the process of RTPCR and the reaction mixture contained extra $33 \mu \mathrm{M}$ of $1 \mathrm{mM}$ Cy5-dCTP.

3.3.3. Assay with Use of IAVchip DNA Microarray. An experimental batch of microarrays was printed on aldehyde substrate (Vantage Aldehyde Slides "CEL Associates") by the method of contact printing of oligonucleotide probes in Nano Print LM 60 (“Arrayit," USA) in accordance with the scheme on Figure 1.

The scheme includes the probes corresponding to hemagglutinins HA1-HA16 and then to neuraminidases NA1-NA9. In addition there is a universal oligonucleotide probe to the region of IAV genes that encode $\mathrm{M}$ and NP proteins shared by all IAV subtypes. Oligonucleotide probes used in the experiments are shown in Table 1.

One microarray slide allows simultaneous analysis of 16 different IAV strains and isolates.

IAVchip DNA microarray was validated with use of IAV reference strains of different subtypes, origin, and biological characteristics [16].

3.3.4. Hybridization. To $1 \mu \mathrm{L}$ of PCR-mixture containing Cy5-cDNA hybridization solution was added; the total volume was brought with $\mathrm{H}_{2} \mathrm{O}$ up to $50 \mu \mathrm{L}$ and heated in the solid-state thermostat at $99^{\circ} \mathrm{C}$ for $2 \mathrm{~min}$ and then cooled in ice for $2 \mathrm{~min}$ and at once applied onto the microarray. In parallel the oligonucleotide probes on the microarray were denatured by boiling of the slide in $\mathrm{H}_{2} \mathrm{O}$ for $1 \mathrm{~min}$ followed by incubation in $96 \%$ ethanol $\left(\mathrm{C}-20^{\circ} \mathrm{C}\right)$ for $1 \mathrm{~min}$. After that the slide was dried by centrifugation at $300 \mathrm{~g}$ for $2 \mathrm{~min}$. Hybridization was performed with use of a frame for 16 subarrays FAST Frame ("Whatman", USA) for $2 \mathrm{~h}$ at $37^{\circ} \mathrm{C}$ and stirring at $250 \mathrm{rpm}$. After hybridization the slide was rinsed in $3 \times$ SSC buffer for $2 \mathrm{~min}$ and in $1 \times$ SSC buffer for $2 \mathrm{~min}$ to remove unbound molecules of the sample and hybridization buffer. After that the frame was removed and the slide was rinsed with water for $2 \mathrm{~min}$. It was dried by centrifugation at $300 \mathrm{~g}$ for $2 \mathrm{~min}$. 
TABLE 1: Oligonucleotide probes for influenza A virus subtyping.

\begin{tabular}{|c|c|c|}
\hline Subtype & Probe & Sequence $\left(5^{\prime} \rightarrow 3^{\prime}\right)^{*}$ \\
\hline \multicolumn{3}{|c|}{ hemagglutinin } \\
\hline \multirow{3}{*}{1} & H1-1 & gaagggagaatgaactattactggacactagtagagccgggagacaa \\
\hline & H1-2 & gtctccctgggggcaatcagyttctggatgtgytcyaatggg \\
\hline & $\mathrm{H} 1-3$ & acaggactaaggaacatcccatccattcaatccagaggtttgtttgg \\
\hline \multirow{4}{*}{2} & $\mathrm{H} 2-1$ & taaggaatgttccccagattgaatcaagaggattgtttggggcaat \\
\hline & $\mathrm{H} 2-2$ & gtcaccgtgactcatgccaaggacattcttgagaaaacgcataatgg \\
\hline & $\mathrm{H} 2-3$ & tatgctacagtagcaggytccetgtcactggcaatcatgata \\
\hline & $\mathrm{H} 2-4$ & tgggatgtcataaattttgagagcactggtaatttaattgcaccagaata \\
\hline \multirow{3}{*}{3} & $\mathrm{H} 3-1$ & atgtgggcctgccararaggcaacattaggtgcaacatttgc \\
\hline & $\mathrm{H} 3-2$ & atgtgggcttgccaaaaaggcaacatcagatgcaacatttgc \\
\hline & $\mathrm{H} 3-3$ & cagcaactgttacccttatgatgtgccggattatgyctccct \\
\hline \multirow{3}{*}{4} & $\mathrm{H} 4-1$ & gcactrcttttagcctttattttgtgggcttgtcagaatggaaacat \\
\hline & $\mathrm{H} 4-2$ & attttgtgggcttgtcagaayggaaacatccggtgccagatttg \\
\hline & $\mathrm{H} 4-3$ & ttccatatcatgcttyttgctcgttgcactrcttttagcctt \\
\hline \multirow{6}{*}{5} & H5-1 & ttgggacatcaacactaaaccagagattggtaccaaraatagctactaga \\
\hline & H5-2 & ttattcaacagtggcragttccctagcactggcaatcatggt \\
\hline & H5-3 & cccaacaataaagagragytacaataataccaaccaagaagatcttttgg \\
\hline & H5-4 & cccaacaataaagaggacctayaacaacaccaatgtagaagaccttttaa \\
\hline & H5-5 & tttatagagggaggatggcagggaatggtagatggttggtatgg \\
\hline & H5-6 & ctagatgtctggacttataatgctgaacttctggttctcatggaaaatga \\
\hline \multirow{3}{*}{6} & H6-1 & cttggtgtgtatcaaattcttgcyatttatagtacggtatcgagcag \\
\hline & H6-2 & caaatccttgcyatttatagtacggtatcgagcagtctrgttttgg \\
\hline & H6-3 & gcaatgggtctttggatgtgttcaaatggttcaatgcartgca \\
\hline \multirow{4}{*}{7} & H7-1 & atgggattggttttcatttgcataaagaatggaaacatgcrgtgcactat \\
\hline & $\mathrm{H} 7-2$ & cttcggggcatcatgtttcatacttctggccattgcaatggg \\
\hline & H7-3 & catcaaaatgcacaaggagarggaactgcagctgactacaaa \\
\hline & $\mathrm{H} 7-4$ & tggtttagcttcggggcatcatgcttcctwcttcttgccattgcaatggg \\
\hline 8 & H8 & atttacagtacagtggcggccagtctytgcttggcaatcctg \\
\hline \multirow{3}{*}{9} & H9-1 & cttacaaaatcctyaccatttattcgactgtcgcctcatctcttgt \\
\hline & H9-2 & gcaatggggtttgctgccttcytrttctgggccatgtccaat \\
\hline & H9-3 & ttctgggccatgtcyaatggatcttgcagatgcaacatttgtat \\
\hline \multirow{2}{*}{10} & H10-1 & cttttggctgtcatcatggggcttgttttcttctgtytgaaaaatggaaa \\
\hline & $\mathrm{H} 10-2$ & gtcatcaattggacyaaggattcaataaccgacatctggacttatcarg \\
\hline \multirow{2}{*}{11} & H11-1 & gatctccatgattctaatgttcgaaacctccatgaaaaggtcagacgaat \\
\hline & H11-2 & tgggcgtgcagyaatggatcatgtagatgtaccatttgcatt \\
\hline 12 & $\mathrm{H} 12$ & tactgctcatgattattgggggtttcattttcggrtgtcaaaatggaaat \\
\hline \multirow{2}{*}{13} & H13-1 & agtgttgtgytagtaggactcatactctctttcatcatgtgggcc \\
\hline & $\mathrm{H} 13-2$ & ataaatatgcttgcagacagaatagatgaygctgtaactgatgta \\
\hline 14 & H14 & tgcatcacccatcaagcgataatgagcaaacggatctctacaagg \\
\hline 15 & H15 & gctgatctgataatagaaagaagaaattcaagtgacatctgttacccagg \\
\hline \multirow{2}{*}{16} & H16-1 & taatgccattgatgaaggagatggttgcttcaatcttcttcacaa \\
\hline & $\mathrm{H} 16-2$ & attcgaaatgggacatataatcatgaggactacaaagaagagtcacaa \\
\hline \multicolumn{3}{|c|}{ Neuraminidase } \\
\hline \multirow{4}{*}{1} & N1-1 & gggttggtcttggccagacggtgctgagttgccattyaccatt \\
\hline & N1-2 & tcctaatggatggacarataccgacagtgatttctcagtgaaacaggatg \\
\hline & N1-3 & tggtcttggccagacggtgctgagttgccvttcaccattgac \\
\hline & N1-4 & caagagtctgaatgtgcatgtgtaaatggytcttgctttactgtaatgac \\
\hline \multirow{4}{*}{2} & $\mathrm{~N} 2-1$ & caagtgtgyatagcatggtccagctcaagttgtcacgatggaaa \\
\hline & $\mathrm{N} 2-2$ & tttggsraccaaacaagtgtgcatagcatggtccagctcaag \\
\hline & $\mathrm{N} 2-3$ & ttttgtggcacttcaggyachtatggaacaggctcatggcct \\
\hline & $\mathrm{N} 2-4$ & tgtgghacytcaggtacatatggaacaggctcatggcctgat \\
\hline
\end{tabular}


TABle 1: Continued.

\begin{tabular}{|c|c|c|}
\hline Subtype & Probe & Sequence $\left(5^{\prime} \rightarrow 3^{\prime}\right)^{*}$ \\
\hline 3 & N3 & agyaatagtatagttactttctgtggaytagacaatgaacctggatcggg \\
\hline 4 & N4 & tgtggtgttaattctgataccacaggttggtcatggccygatggc \\
\hline 5 & N5 & ttttgtggtgtttcmagtgaggtcccaggrtggtcctgggatgatgg \\
\hline \multirow{3}{*}{6} & N6-1 & tcatgccatgacggcatctcaagaatgtcratctgcatgtca \\
\hline & N6-2 & gagcgattrggatcatggtcctggcatgatggtgctgaratc \\
\hline & N6-3 & gagcgattgggatcktggtcatggcatgatggggctgaaatc \\
\hline \multirow{2}{*}{7} & N7-1 & gttgaaggatgggtagtggtggcyaaggacaatgccataagatt \\
\hline & N7-2 & cagttgggtccggttccttccccgatggggcacaratccaat \\
\hline \multirow{3}{*}{8} & N8-1 & atatggacctcwagyagctccattgtgatgtgtggagtagaycat \\
\hline & N8-2 & tgagtgtagaaatagggcaatcacccaatgtgtaccaggcaaggt \\
\hline & N8-3 & tggtcrtggcacgatggagctattcttccytttgacatcgat \\
\hline 9 & N9 & atgtgttccagyacagaattcctkggacaatggaactggcctgat \\
\hline \multicolumn{3}{|c|}{ M2-protein } \\
\hline & M2-1 & gcagartgctgtggatgttgacgatrgtcattttgtcaacatag \\
\hline & M2-2 & cctatcagaaacgaatgggggtgcagatgcaacgattcaagtga \\
\hline & M2-3 & ccttctacggaaggagtrccwgagtctatgagggaagaatatcg \\
\hline \multicolumn{3}{|c|}{ NP protein } \\
\hline & NP-1 & acgaaaaggcaacgaacccgatcgtgccttcctttgacatga \\
\hline & NP-2 & atgagtaatgaaggdtcttatttcttcggagacaatgcagargag \\
\hline
\end{tabular}

3.3.5. Scanning. The microarrays were scanned in InnoScan710AL ("Innopsys," France) with $5 \mu \mathrm{m}$ resolution. Fluorescent scanning was performed at wavelengths $532 \mathrm{~nm}$ and $635 \mathrm{~nm}$. The resulted images were processed with use of the software Mapixver. 5.5.0 ("Innopsys", France).

3.4. Virus Isolation in Chicken Embryos. Virus isolation was performed in 9-10-day chicken embryos (CE) following the standard procedures where influenza virus production was confirmed by identification in hemagglutination inhibition (HI) test [19].

3.5. Real-Time RT-PCR. Real-time RT-PCR was performed by method of "TaqMan" with use of Light Cycler 2.0 manufactured by Roche Company. Real-time RT-PCR for detection of $\mathrm{H} 1 \mathrm{~N} 1$ and $\mathrm{H} 3 \mathrm{~N} 2$ subtypes was performed using primers and conditions described by Schweiger et al. (2000) [20], for detection of H3N8 using primers and conditions described by Lu et al. (2009) [21] and for detection of H5N1 using primers and conditions according to WHO guidelines (2007) [22].

3.6. RT-PCR. RT-PCR for detection of H1N1 and $\mathrm{H} 3 \mathrm{~N} 2$ subtypes was performed according to Schweiger et al. (2000) [20], for detection of H3N8 according to Lu et al. (2009) and Chervyakova et al. (2014) [21, 23], and for detection of H5N1 subtype according to WHO guidelines (2007) [22].

Specific regions of IAV cDNA were produced in GeneAmp PCR 9700, Applied Biosystems. Table 2 shows oligonucleotide primers that were used in the study.

3.7. Statistical Analysis. True positive (TP), true negative (TN), false positive (FP), and false negative (FN) results of the assays were used to assess effectiveness of laboratory tests.
The following calculations were used: sensitivity $(\mathrm{SN})=$ $(\mathrm{TP} / \mathrm{TP}+\mathrm{FN})$, specificity $(\mathrm{SP})=(\mathrm{TN} / \mathrm{TN}+\mathrm{FP})$, Positive Predictive Value $(\mathrm{PPV})=(\mathrm{TP} / \mathrm{TP}+\mathrm{FP})$, Negative Predictive Value $(\mathrm{NPV})=(\mathrm{TN} / \mathrm{TN}+\mathrm{FN})$, and diagnostic effectiveness $(\mathrm{DE})=(\mathrm{TP}+\mathrm{TN} / \mathrm{TP}+\mathrm{FP}+\mathrm{FN}+\mathrm{TN})[24]$.

$95 \%$ confidence intervals $(95 \% \mathrm{CI})$ were calculated according to van Engelsdorp et al. [24].

\section{Results}

4.1. Detection of Influenza A Virus in Clinical Specimens. Diagnostic effectiveness of IAVchip DNA microarray in comparison to the virus isolation in chicken embryos, real-time RT-PCR, and RT-PCR for IAV detection and subtyping was evaluated by testing 185 clinical specimens from humans, animals, and birds.

"True" state of infection is determined by the most accurate diagnostic method that is called "gold standard." In diagnostics and identification of the influenza infection virus isolation in chicken embryos followed by identification in $\mathrm{HI}$ is the "gold standard" [19].

Table 3 shows the results of detecting IAV in clinical specimens by various methods in comparison to virus isolation in chicken embryos.

In our experiments IAV was isolated by the method of virus isolation in chicken embryos from 98 (52.97\%) specimens out of 185 clinical samples taken from humans, animals, and birds. The rest of samples (87, i.e., 47.03\%) showed negative result. The same picture was observed in the experiments aimed at IAV isolation with use of IAVchip DNA microarray. In these tests the influenza A virus was detected in 98 (52.97\%) samples. At the same time the method of realtime PCR displayed the presence of IAV only in 96 (51.89\%) 


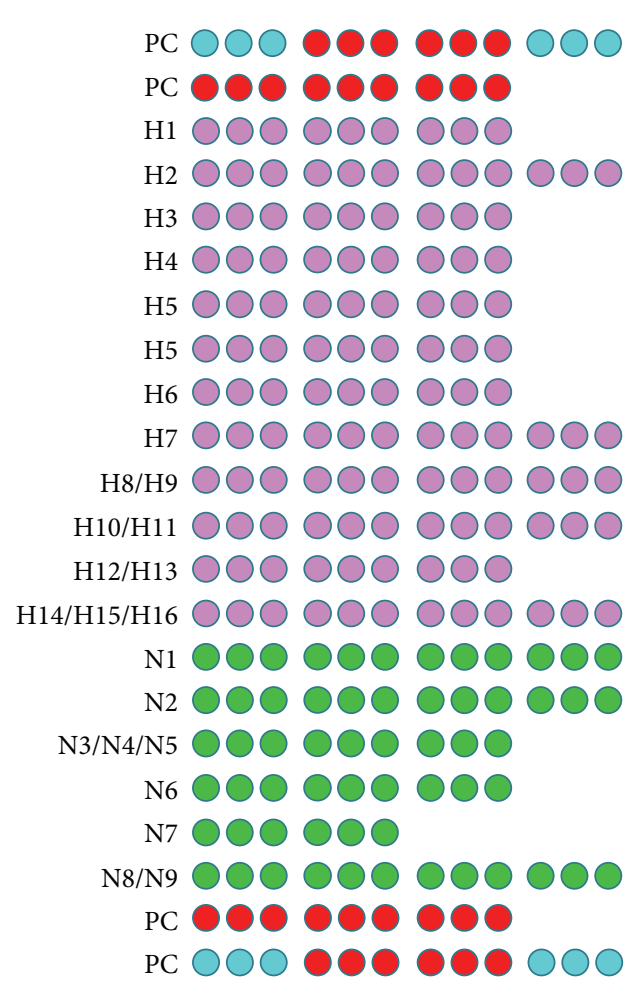

\begin{tabular}{lc|c|c|cc}
\hline \multicolumn{1}{c}{1} & \multicolumn{1}{c}{3} & \multicolumn{1}{c}{4} \\
\hline 1 & NNM & NP-1 & NP-2 & NNM & PC \\
2 & M2-1 & M2-2 & M2-3 & BUF & PC \\
3 & H1-1 & H1-2 & H1-3 & BUF & H1 \\
4 & H2-1 & H2-2 & H2-3 & H2-4 & H2 \\
5 & H3-1 & H3-2 & H3-3 & BUF & H3 \\
6 & H4-1 & H4-2 & H4-3 & BUF & H4 \\
7 & H5-1 & H5-2 & H5-3 & BUF & H5 \\
8 & H5-4 & H5-5 & H5-6 & BUF & H5 \\
9 & H6-1 & H6-2 & H6-3 & BUF & H6 \\
10 & H7-1 & H7-2 & H7-3 & H7-4 & H7 \\
11 & H8 & H9-1 & H9-2 & H9-3 & H8/H9 \\
12 & H10-1 & H10-2 & H11-1 & H11-2 & H10/H11 \\
13 & H12 & H13-1 & H13-2 & BUF & H12/H13 \\
14 & H14 & H15 & H16-1 & H16-2 & H14/H15/H16 \\
15 & N1-1 & N1-2 & N1-3 & N1-4 & N1 \\
16 & N2-1 & N2-2 & N2-3 & N2-4 & N2 \\
17 & N3 & N4 & N5 & BUF & N3/N4/N5 \\
18 & N6-1 & N6-2 & N6-3 & BUF & N6 \\
19 & N7-1 & N7-2 & BUF & BUF & N7 \\
20 & N8-1 & N8-2 & N8-3 & N9 & N8/N9 \\
21 & M2-1 & M2-2 & M2-3 & BUF & PC \\
22 & NNM & NP-1 & NP-2 & NNM & PC \\
\hline
\end{tabular}

FIGURE 1: Scheme of the model IAVchip microarray for influenza A virus subtyping. H: hemagglutinin, N: neuraminidase, PC: positive control, BUF: 1xMicroarray printing buffer Arrayit, and NNM: hybridization control.

specimens, and the method of RT-PCR merely in $71(38.38 \%)$ samples. Percentage of false positive results in the tests with use of IAVchip DNA microarray and in the real-time PCR was $0.54 \%$ and $2.16 \%$ in RT-PCR. Use of IAVchip DNA microarray did not show false negative responses, while real-time PCR and RT-PCR displayed $1.08 \%$ and $1.62 \%$, respectively.

4.2. Comparison of Various Tests for Influenza A Diagnosis. Sensitivity and specificity of each test were determined to evaluate effectiveness and reliability of influenza A diagnostic tests.

Sensitivity and specificity indices, as well as positive and negative prognostic values of the developed IAVchip DNA microarray, of RT-PCR and real-time RT-PCR with 95\% confidence interval are displayed in Table 4.

Method of virus isolation in chicken embryos being used as a standard, IAVchip DNA microarray displayed 100\% diagnostic sensitivity and $98.85 \%$ diagnostic specificity within the range $97.35-100 \%$ of $95 \%$ CI. Diagnostic sensitivity of IAVchip DNA microarray (100\%) exceeds the same parameter of realtime RT-PCR (97.96\%) within the range $95.96 \%-99.96 \%$ of 95\% CI and of RT-PCR (95.95\%) within the range $93.15 \%-$ $98.75 \%$ of $95 \%$ CI.

In influenza A diagnosis IAVchip DNA microarray and real-time PCR demonstrated equal diagnostic specificity (98.85\%), while the same characteristic of RT-PCR was 96.40\%. Diagnostic specificity of IAVchip DNA microarray and real-time PCR were in the range of the confidence intervals of the RT-PCR specificity. The limit of the $95 \%$ confidence intervals for RT-PCR was $93.70 \%-99.10 \%$.

PPV and NPV values of IAVchip DNA microarray were 98.99\% and $100 \%$, respectively. PPV and NPV of the real-time RT-PCR were also in the range of the estimated confidence intervals.

4.3. Influenza A Virus Subtyping with Use of IAVchip DNA Microarray. IAVchip DNA microarray, real-time RT-PCR, and RT-PCR were used to subtype IAV from 98 samples proved to be positive by the method of the virus isolation in chicken embryos. The IAV were subtyped by the methods of RT-PCR and real-time RT-PCR in 3 steps: identification of the virus (step 1), detection of HA (step 2), and NA (step 3). IAVchip DNA microarray was used to subtype simultaneously the tested IAV with probes specific to hemagglutinin 1-16 and neuraminidase 1-9 genes. Table 5 shows examples of IAV subtyping with the help of IAVchip DNA microarray in the form of histograms.

As Table 5 shows IAVchip DNA microarray makes possible simultaneous IAV subtyping in the tested samples. Possibility in principle to use the DNA microarray for diagnosis and subtyping of various influenza viruses has been demonstrated by examples of seasonal A/H1N1 and $\mathrm{A} / \mathrm{H} 3 \mathrm{~N} 2$ viruses, equine influenza $\mathrm{A} / \mathrm{H} 3 \mathrm{~N} 8$ virus, and highly pathogenic avian influenza $\mathrm{A} / \mathrm{H} 5 \mathrm{~N} 1$ virus. In the assay of the samples the value of specific fluorescence reliably exceeded the value of the background fluorescence $(P<0.05)$. 
TABLE 2: Oligonucleotide primers and probes for influenza A virus typing and subtyping in real-time PCR and RT-PCR.

\begin{tabular}{|c|c|c|}
\hline Influenza virus type/subtype & Primer or probes & Sequence \\
\hline \multirow{3}{*}{ A } & AM-151 & CATGGAATGGCTAAAGACAAGACC \\
\hline & AM-397 & AAGTGCACCAGCAGAATAACTGAG \\
\hline & Probe AM-245 & CTGCAGCGTAGACGCTTTGTCCAAAATG \\
\hline \multicolumn{3}{|l|}{$\mathrm{A} / \mathrm{H} 1 \mathrm{~N} 1$} \\
\hline \multirow{3}{*}{$\mathrm{A} / \mathrm{H} 1$} & HA1-583 & GGTGTTCATCACCCGTCTAACAT \\
\hline & HA1-895 & GTGTTTGACACTTCGCGTCACAT \\
\hline & Probe HA1-783 & TGCCTCAAATATTATTGTGTCCCCGGGT \\
\hline \multirow{3}{*}{$\mathrm{A} / \mathrm{N} 1$} & NA1-1078 & ATGGTAATGGTGTTTGGATAGGAAG \\
\hline & NA1-1352 & AATGCTGCTCCCACTAGTCCAG \\
\hline & Probe NA1-1138 & TGATTTGGGATCCTAATGGATGGACAG \\
\hline \multicolumn{3}{|l|}{ A/H3N2 } \\
\hline \multirow{3}{*}{$\mathrm{A} / \mathrm{H} 3$} & HA3-115 & GCTACTGAGCTGGTTCAGAGTTC \\
\hline & HA3-375 & GAAGTCTTCATTGATAAACTCCAG \\
\hline & Probe HA3-208 & CTATTGGGAGACCCTCATTGTGATGG \\
\hline \multirow{3}{*}{$\mathrm{A} / \mathrm{N} 2$} & NA2-560 & AAGCATGGCTGCATGTTTGTG \\
\hline & NA2-858 & ACCAGGATATCGAGGATAACAGGA \\
\hline & Probe NA2-821 & TGCTGAGCACTTCCTGACAATGGGCT \\
\hline \multicolumn{3}{|l|}{$\mathrm{A} / \mathrm{H} 3 \mathrm{~N} 8$} \\
\hline \multirow{3}{*}{$\mathrm{A} / \mathrm{H} 3$} & EqFlu HA3 F & TCACATGGACAGGTGTCACTCA \\
\hline & EqFlu HA3 R & GGCTGATCCCCTTTTGCA \\
\hline & EqFlu HA3 Pr & AACGGAAGAAGTGGAGC \\
\hline \multirow{2}{*}{$\mathrm{A} / \mathrm{N} 8$} & N8Eq-F30 & TGG ATC TGC ATC ATT GGG GA \\
\hline & N8eq-R535 & CTG ACC ATG CCA CCG ATT CA \\
\hline \multicolumn{3}{|l|}{$\mathrm{A} / \mathrm{H} 5 \mathrm{~N} 1$} \\
\hline \multirow{4}{*}{$\mathrm{A} / \mathrm{H} 5$} & H5HA-205-227v2-F & CGATCTAGAYGGGGTGAARCCTC \\
\hline & H5HA-326-302v2-R & ССТTCTCCACTATGTANGACCATTC \\
\hline & H5-Probe-239-RVa & FAM-AGCCAYCCAGCTACRCTACA-MGB \\
\hline & H5-Probe-239-RVb & FAM-AGCCATCCCGCAACACTACA-MGB \\
\hline \multirow{3}{*}{$\mathrm{A} / \mathrm{N} 1$} & N1-For-474-502 & TAYAACTCAAGGTTTGAGTCTGTYGCTTG \\
\hline & N1-Rev-603-631 & ATGTTRTTCCTCCAACTCTTGATRGTGTC \\
\hline & N1-Probe-501-525 & FAM-TCAGCRAGTGCYTGCCATGATGGCAMGB \\
\hline
\end{tabular}

AM: $M$ gene of influenza A viruses.

$\mathrm{HA1}, \mathrm{HA} 3$, and HA5: HA gene of influenza A viruses of subtypes $\mathrm{H} 1, \mathrm{H} 3$, and $\mathrm{H} 5$, respectively.

NA, NA2, and NA8: NA gene of influenza A viruses of subtypes N1, N2, and N8, respectively.

TABLE 3: Comparison of the results of our test using IAVchip DNA-microarray with the results of real-time RT-PCR and RT-PCR.

\begin{tabular}{lcccc}
\hline \multirow{2}{*}{ Result } & & & Test & \\
& RT-PCR & Real-time PCR & IAVchip DNA microarray & Virus isolation in chicken embryos \\
\hline Positive & $71(38.38 \%)$ & $96(51.89 \%)$ & $98(52.97 \%)$ & $98(52.97 \%)$ \\
False positive & $4(2.16 \%)$ & $1(0.54 \%)$ & $1(0.54 \%)$ & 0 \\
False negative & $3(1.62 \%)$ & $2(1.08 \%)$ & 0 & 0 \\
Negative & $107(57.84 \%)$ & $86(46.49 \%)$ & $86(46.49 \%)$ & $87(47.03 \%)$ \\
\hline Total & $185(100 \%)$ & $185(100 \%)$ & $185(100 \%)$ & $185(100 \%)$ \\
\hline
\end{tabular}

Moreover, $\mathrm{M}$ and NP genes were reliably detected in all samples.

The results of comparative analysis of samples with use of IAVchip DNA microarray, as well as by real-time RT-PCR and RT-PCR, are shown in Table 6.
The findings of the study showed that by use of IAVchip DNA microarray influenza viruses were detected in all 98 samples; $36.73 \%$ of them were subtyped as influenza A virus $\mathrm{A} / \mathrm{H} 1 \mathrm{~N} 1,40.82 \%$ as $\mathrm{A} / \mathrm{H} 3 \mathrm{~N} 2,14.29 \%$ as $\mathrm{A} / \mathrm{H} 3 \mathrm{~N} 8$, and $8.16 \%$ as $\mathrm{A} / \mathrm{H} 5 \mathrm{~N} 1$. 
TABle 4: Comparative diagnostic value of different tests in influenza A diagnosis.

\begin{tabular}{lccr}
\hline Result, \% & RT-PCR & Test & Real-time RT-PCR \\
\hline SN, 95\% CI & $95.95(93.15-98.75)$ & $97.96(95.96-99.96)$ & 100 \\
SP, 95\% CI & $96.40(93.70-99.10)$ & $98.85(97.35-100)$ & $98.85(97.35-100)$ \\
PPV, 95\% CI & $94.67(91.37-97.97)$ & $98.97(97.57-100)$ & $98.99(97.59-100)$ \\
NPV, 95\% CI & $97.27(94.97-99.57)$ & $97.73(95.63-99.83)$ & 100 \\
\hline
\end{tabular}

SN: sensitivity; SP: specificity; PPV: Positive Predictive Value; NPV: Negative Predictive Value; 95\% CI: 95\% confidence interval.

In the same samples influenza viruses were detected and subtyped by RT-PCR as follows: in $24.49 \%$ of samples as $\mathrm{A} / \mathrm{H} 1 \mathrm{~N} 1$, in $31.63 \%$ as $\mathrm{A} / \mathrm{H} 3 \mathrm{~N} 2$, in $11.22 \%$ as $\mathrm{A} / \mathrm{H} 3 \mathrm{~N} 8$, and in $5.10 \%$ as $\mathrm{A} / \mathrm{H} 5 \mathrm{~N} 1$. The results of RT-PCR were positive in $72.45 \%$ samples; in $27.55 \%$ they were negative.

In the same 98 samples real-time RT-PCR detected and subtyped the following influenza viruses: A/H1N1 in $35.71 \%$, $\mathrm{A} / \mathrm{H} 3 \mathrm{~N} 2$ in $39.80 \%, \mathrm{~A} / \mathrm{H} 3 \mathrm{~N} 8$ in $14.29 \%$, and $\mathrm{A} / \mathrm{H} 5 \mathrm{~N} 1$ in $8.16 \%$ of samples. Real-time RT-PCR diagnosed influenza A virus in $97.96 \%$ of all assayed samples and showed negative result in $2.04 \%$ of samples.

\section{Discussion}

IAV genome variability begets a very important diagnostic problem that consists in need of rapid and accurate method of diagnosis not only of existing but of emerging viruses for efficient influenza surveillance at the global level [25, 26].

Biological microarrays are developed today in many countries. Biochips for diagnosis of the human influenza of subtypes H1N1, H3N2, including H5N1, are set forth [27]. It should be noted that various approaches were used. MChip was developed with use of probes only to $M$ gene of the influenza virus, but further on it was supplemented with probes to hemagglutinin and neuraminidase genes (FluChip) [11]. The microarrays proposed by Huang et al. [1] and Teo et al. [28] contain probes to the hemagglutinin and nonstructural protein genes of the influenza $B$ virus. These diagnostic microarrays can be used only for diagnosis of the seasonal influenza because reassortment and mutations so characteristic of the influenza virus result in emergence of new subtypes that are isolated both from animal and bird populations and from humans.

There are also microarrays for identification of all IAV subtypes by both hemagglutinin and neuraminidase genes. Xueqing et al. [29] used 52 oligonucleotide probes for typing IAV. The viral cDNA was amplified in multiplex PCR with 25 pairs of primers. Multiplex PCR for amplification of the influenza virus cDNA with use of 25 pairs of primers was performed by Han et al. [14]. The component structure was optimized for performance of the reaction in 4 tubes. Quan et al. [30] developed microarray GreeneChipResp that enables identifying 20 respiratory viral infections apart from typing all subtypes of IAV.

On the basis of the oligonucleotide microarray IAVchip that allows detecting and subtyping IAV has been developed at the RIBSP [15]. This microarray is universal for all subtypes of IAV and when necessary enables screening the IAV not only by HA and NA, but by M and NP genes as well.

Diagnostic effectiveness (DE) of the test expressed as percentage ratio of the number of true test results to the total number of findings for IAVchip DNA microarray was $99.45 \%$, for the real-time PCR and RT-PCR; it was $98.38 \%$ and $96.22 \%$, respectively.

The majority of authors propose in their papers using microarrays for the human influenza viruses $[9,11,31]$, whereas our biological chip is designed to identify influenza viruses from humans animals and birds.

Analysis of these data shows that IAVchip DNA microarray for IAV diagnosis and identification has the advantage over the other tests in effectiveness and is more rapid owing to possibility to detect and subtype several influenza A viruses simultaneously in one experiment.

It should be noted that in the course of testing clinical specimens with use of IAVchip DNA microarray in one reaction both influenza $A$ virus identification and subtyping of tens of viruses take place during 8-10 hours. Subtype information is especially important, for example, in the South-Eastern Asia, where subtypes A/H3N2, A/H1N1, and $\mathrm{A} / \mathrm{H} 5 \mathrm{~N} 1$ can simultaneously circulate in a body.

So, possible usage of the microarray in clinical practice for influenza A diagnosis and subtyping of the viruses isolated both from humans and from animals and birds is shown.
Abbreviations
IAV: Influenza A virus
HA: Hemagglutinin
NA: Neuraminidase
TP: True positive
TN: True negative
FP: $\quad$ False positive
FN: $\quad$ False negative
SN: $\quad$ Sensitivity
SP: $\quad$ Specificity
PPV: $\quad$ Positive Predictive Value
NPV: Negative Predictive Value
DE: Diagnostic effectiveness
95\% CI: 95\% confidence intervals.

\section{Ethical Approval}

Ethical approval was not required. 


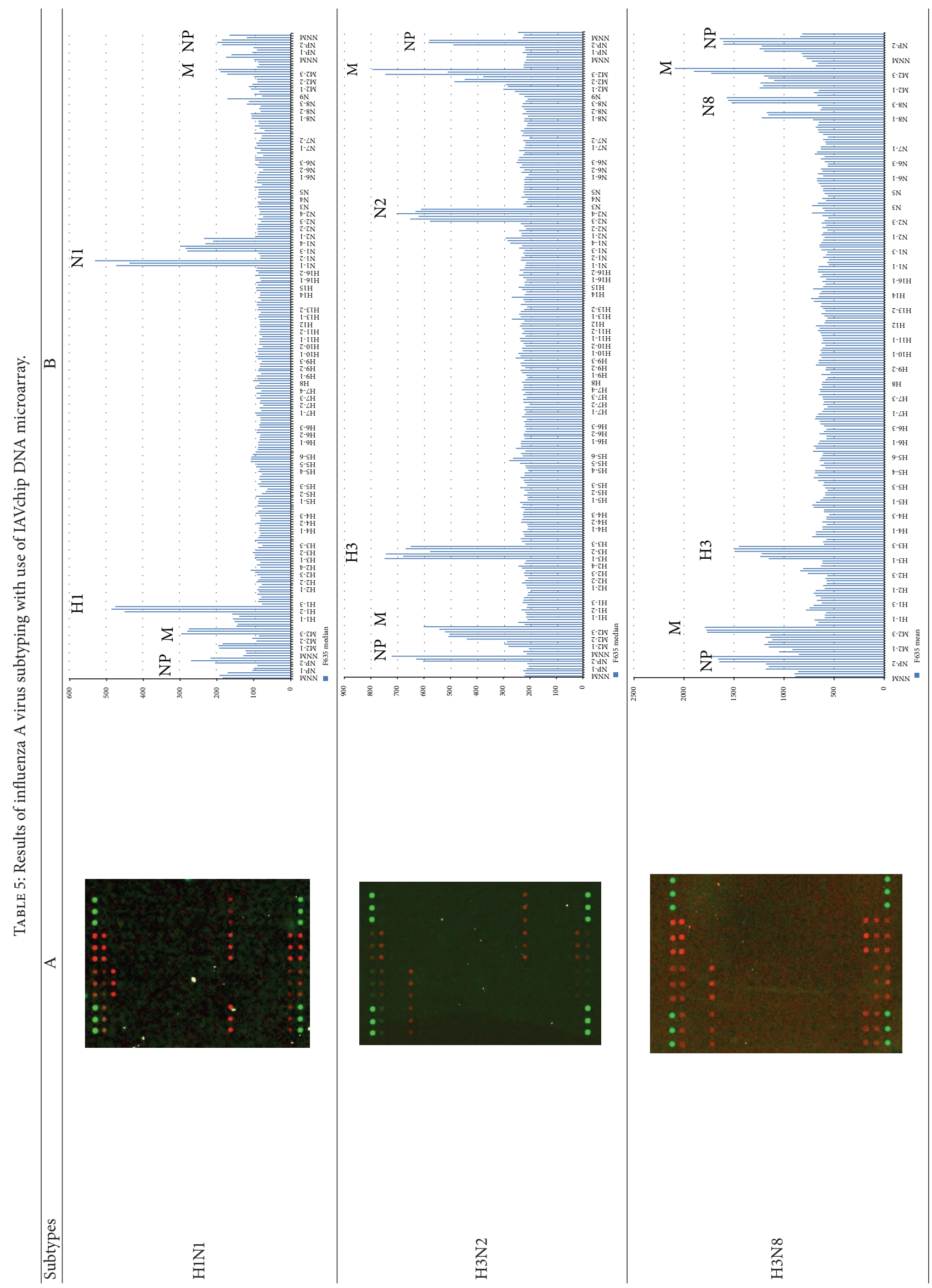




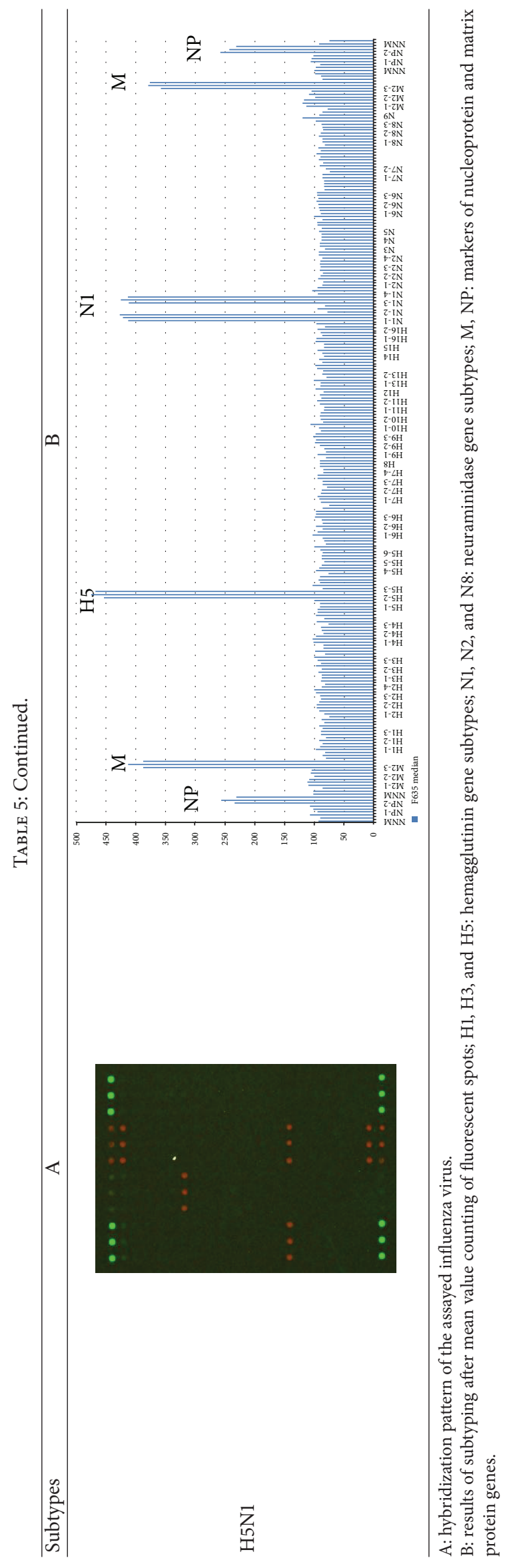


TABLE 6: Results of detecting influenza A virus subtypes in clinical specimens by various tests.

\begin{tabular}{|c|c|c|c|c|c|c|c|}
\hline \multirow{4}{*}{ Subtype } & \multicolumn{7}{|c|}{ Test } \\
\hline & \multicolumn{3}{|c|}{ Reverse transcription PCR } & \multicolumn{3}{|c|}{ Real-time RT-PCR } & \multirow{3}{*}{$\begin{array}{c}\text { IAVchip DNA microarray } \\
\text { Step } 1 \\
\text { Type A, H, N }\end{array}$} \\
\hline & Step 1 & Step 2 & Step 3 & Step 1 & Step 2 & Step 3 & \\
\hline & Type A & $\mathrm{H}$ & $\mathrm{N}$ & Type A & $\mathrm{H}$ & $\mathrm{N}$ & \\
\hline H1N1 & $24(24.49 \%)$ & $24(24.49 \%)$ & $24(24.49 \%)$ & $35(35.71 \%)$ & $35(35.71 \%)$ & $35(35.71 \%)$ & $36(36.73 \%)$ \\
\hline $\mathrm{H} 3 \mathrm{~N} 2$ & $31(31.63 \%)$ & $31(31.63 \%)$ & $31(31.63 \%)$ & $39(39.80 \%)$ & $39(39.80 \%)$ & $39(39.80 \%)$ & $40(40.82 \%)$ \\
\hline $\mathrm{H} 3 \mathrm{~N} 8$ & $11(11.22 \%)$ & $11(11.22 \%)$ & $11(11.22 \%)$ & $14(14.29 \%)$ & $14(14.29 \%)$ & N/A & $14(14.29 \%)$ \\
\hline $\mathrm{H} 5 \mathrm{~N} 1$ & $5(5.10 \%)$ & $5(5.10 \%)$ & $5(5.10 \%)$ & $8(8.16 \%)$ & $8(8.16 \%)$ & $8(8.16 \%)$ & $8(8.16 \%)$ \\
\hline Positive & $71(72.45 \%)$ & $71(72.45 \%)$ & $71(72.45 \%)$ & $96(97.96 \%)$ & $96(97.96 \%)$ & $82(83.67 \%)$ & $98(100 \%)$ \\
\hline
\end{tabular}

Note: 98 specimens were assayed.

\section{Conflict of Interests}

The authors declare that there is no conflict of interests regarding the publication of this paper.

\section{References}

[1] Y. Huang, H. Tang, S. Duffy et al., "Multiplex assay for simultaneously typing and subtyping influenza viruses by use of an electronic microarray," Journal of Clinical Microbiology, vol. 47, no. 2, pp. 390-396, 2009.

[2] J. M. Daly, A. S. Blunden, S. MacRae et al., "Transmission of equine influenza virus to english foxhounds," Emerging Infectious Diseases, vol. 14, no. 3, pp. 461-464, 2008.

[3] P. C. Crawford, E. J. Dubovi, W. L. Castleman et al., "Epidemiology: transmission of equine influenza virus to dogs," Science, vol. 310, no. 5747, pp. 482-485, 2005.

[4] B. A. Cunha, "Influenza: historical aspects of epidemics and pandemics," Infectious Disease Clinics of North America, vol. 18, no. 1, pp. 141-155, 2004.

[5] M. J. Ma, X.-X. Yang, X. Xia et al., "omparison of commercial influenza A virus assays in detecting avian influenza H7N9 among poultry cloacal swabs," Journal of Clinical Virology, vol. 59, no. 4, pp. 242-245, 2014.

[6] M. Du Ry van Beest Holle, A. Meijer, M. Koopmans, and C. M. de Jager, "Outbreak report: human-to-human transmission of avian influenza A/H7N7, The Netherlands, 2003," Eurosurveillance, vol. 10, no. 12, p. 584, 2005.

[7] V. C. C. Cheng, J. F. W. Chan, X. Wen et al., "Infection of immunocompromised patients by avian H9N2 influenza A virus," Journal of Infection, vol. 62, no. 5, pp. 394-399, 2011.

[8] F. C. K. Li, B. C. K. Choi, T. Sly, and A. W. P. Pak, "Finding the real case-fatality rate of $\mathrm{H} 5 \mathrm{~N} 1$ avian influenza," Journal of Epidemiology and Community Health, vol. 62, no. 6, pp. 555$559,2008$.

[9] N. Kessler, O. Ferraris, K. Palmer, W. Marsh, and A. Steel, "Use of the DNA flow-thru chip, a three-dimensional biochip, for typing and subtyping of influenza viruses," Journal of Clinical Microbiology, vol. 42, no. 5, pp. 2173-2185, 2004.

[10] M. J. Lodes, D. Suciu, M. Elliott et al., "Use of semiconductorbased oligonucleotide microarrays for influenza A virus subtype identification and sequencing," Journal of Clinical Microbiology, vol. 44, no. 4, pp. 1209-1218, 2006.

[11] M. B. Townsend, E. D. Dawson, M. Mehlmann et al., "Experimental evaluation of the FluChip diagnostic microarray for influenza virus surveillance," Journal of Clinical Microbiology, vol. 44, no. 8, pp. 2863-2871, 2006.
[12] S. Sengupta, K. Onodera, A. Lai, and U. Melcher, "Molecular detection and identification of influenza viruses by oligonucleotide microarray hybridization," Journal of Clinical Microbiology, vol. 41, no. 10, pp. 4542-4550, 2003.

[13] E. E. Fesenko, D. E. Kireyev, D. A. Gryadunov et al., "Oligonucleotide microchip for subtyping of influenza A virus," Influenza and Other Respiratory Viruses, vol. 1, no. 3, pp. 121-129, 2007.

[14] X. Han, X. Lin, B. Liu et al., "Simultaneously subtyping of all influenza A viruses using DNA microarrays," Journal of Virological Methods, vol. 152, no. 1-2, pp. 117-121, 2008.

[15] A. V. Vasin, N. T. Sandybaev, M. A. Plotnikova et al., "Multisegment one-step RT-PCR fluorescent labeling of influenza A virus genome for use in diagnostic microarray applications," Journal of Physics, vol. 291, no. 1, 2011.

[16] A. V. Vasin, N. T. Sandybayev, M. A. Plotnikova, S. A. Klotchenko, O. V. Chervyakova, and V. M. Strochkov, "Diagnostic oligonucleotide microchip for detection and subtyping of the human and animal influenza A viruses," Virology Issues, vol. 5, pp. 32-37, 2013 (Russian).

[17] G. G. Onishchenko, O. I. Kiselev, and A. A. Sominina, Influenza surveillance and control amplification as the most significant element in preparation for seasonal epidemics and a recurrent pandemic. Collection of methodological recommendations compiled on the basis of WHO materials. MoscowSt.Petersburg, pp. 42-48, 2004.

[18] E. Hoffmann, J. Stech, Y. Guan, R. G. Webster, and D. R. Perez, "Universal primer set for the full-length amplification of all influenza A viruses," Archives of Virology, vol. 146, no. 12, pp. 2275-2289, 2001.

[19] World Organization for Animal Health (OIE), Avian Influenza: Manual of Diagnostic Tests and Vaccines for Terrestrial Animals, chapter 2-4, OIE, Paris, France, 2008.

[20] B. Schweiger, I. Zadow, R. Heckler, H. Timm, and G. Pauli, "Application of a fluorogenic PCR assay for typing and subtyping of influenza viruses in respiratory samples," Journal of Clinical Microbiology, vol. 38, no. 4, pp. 1552-1558, 2000.

[21] Z. Lu, T. M. Chambers, S. Boliar et al., "Development and evaluation of one-step TaqMan real-time reverse transcriptionPCR assays targeting nucleoprotein, matrix, and hemagglutinin genes of equine influenza virus," Journal of Clinical Microbiology, vol. 47, no. 12, pp. 3907-3913, 2009.

[22] WHO, Recommendations and Laboratory Procedures for Detection of Avian Influenza A(H5N1) Virus in Specimens from Suspected Human Cases, WHO, 2007. 
[23] O. V. Chervyakova, V. M. Strochkov, E. T. Tailakova et al., "Recombinant strain A/HK/Otar/6:2/2010 (H3N8) for development of a live intranasal equine influenza vaccine," Journal of Equine Veterinary Science, vol. 34, pp. 749-758, 2014.

[24] D. van Engelsdorp, E. Lengerich, A. Spleen et al., "Standard epidemiological methods to understand and improve Apis mellifera health," Journal of Apicultural Research, vol. 52, no. 1, 2013.

[25] C. Debouck and P. N. Goodfellow, "DNA microarrays in drug discovery and development," Nature Genetics, vol. 21, no. 1, pp. 48-50, 1999.

[26] J. A. McCullers, G. C. Wang, S. He, and R. G. Webster, "Reassortment and insertion-deletion are strategies for the evolution of influenza B viruses in nature," Journal of Virology, vol. 73, no. 9, pp. 7343-7348, 1999.

[27] E. D. Dawson, C. L. Moore, J. A. Smagala et al., "MChip: a tool for influenza surveillance," Analytical Chemistry, vol. 78, no. 22, pp. 7610-7615, 2006.

[28] J. Teo, P. D. Pietro, F. S. Biagio et al., "VereFlu: an integrated multiplex RT-PCR and microarray assay for rapid detection and identification of human influenza $A$ and $B$ viruses using lab-onchip technology," Archives of Virology, vol. 156, no. 8, pp. 13711378, 2011.

[29] H. Xueqing, L. Xiangmei, H. Yihong et al., "Oligonucleotide microarray for subtyping avian influenza virus," Wei Sheng $W u$ Xue Bao, vol. 48, no. 9, pp. 1241-1249, 2008.

[30] P.-L. Quan, G. Palacios, O. J. Jabado et al., "Detection of respiratory viruses and subtype identification of influenza A viruses by greenechipresp oligonucleotide microarray," Journal of Clinical Microbiology, vol. 45, no. 8, pp. 2359-2364, 2007.

[31] M. Mehlmann, A. B. Bonner, J. V. Williams et al., "Comparison of the MChip to viral culture, reverse transcription-PCR, and the QuickVue influenza $\mathrm{A}+\mathrm{B}$ test for rapid diagnosis of influenza," Journal of Clinical Microbiology, vol. 45, pp. 12341237, 2007. 

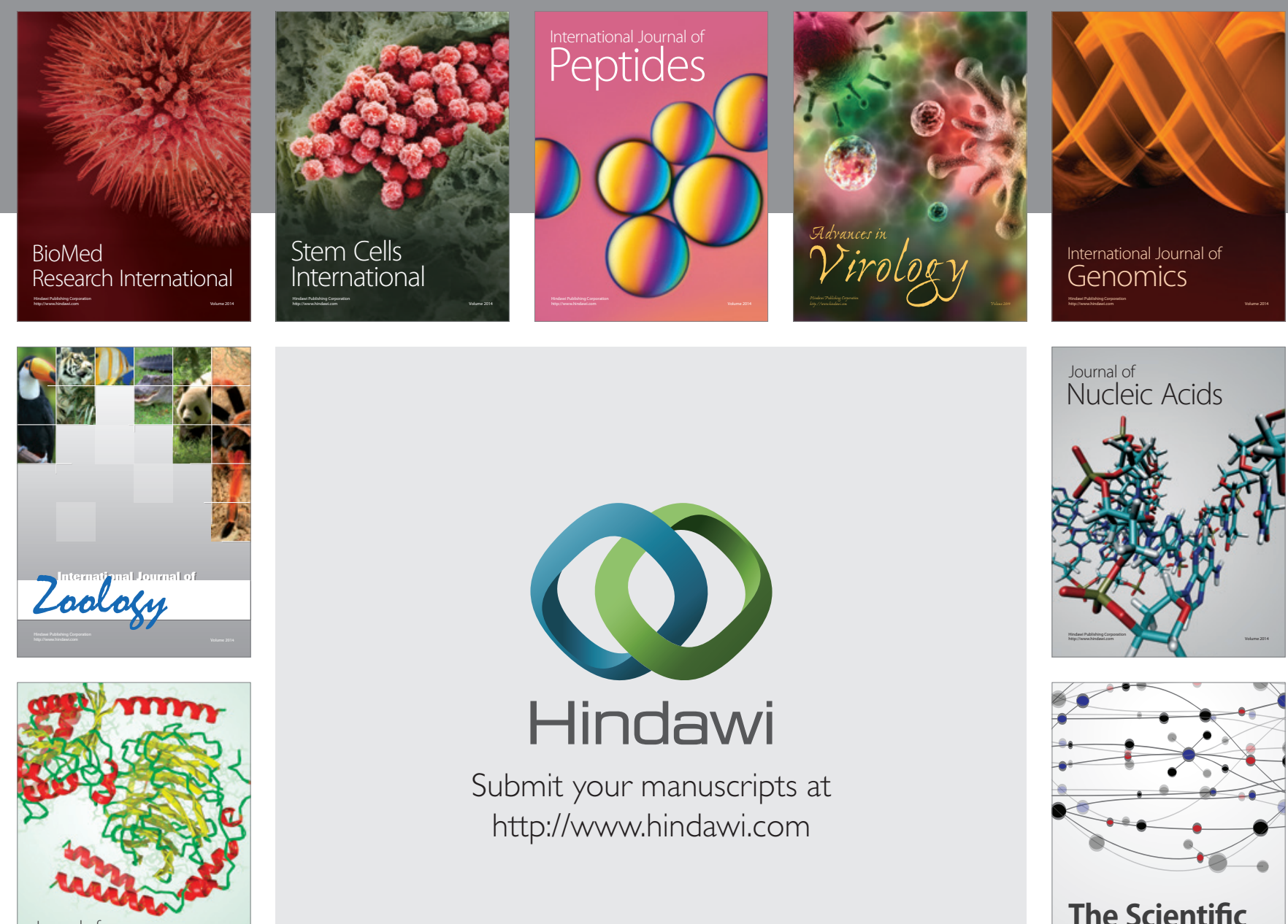

Submit your manuscripts at

http://www.hindawi.com

Journal of
Signal Transduction
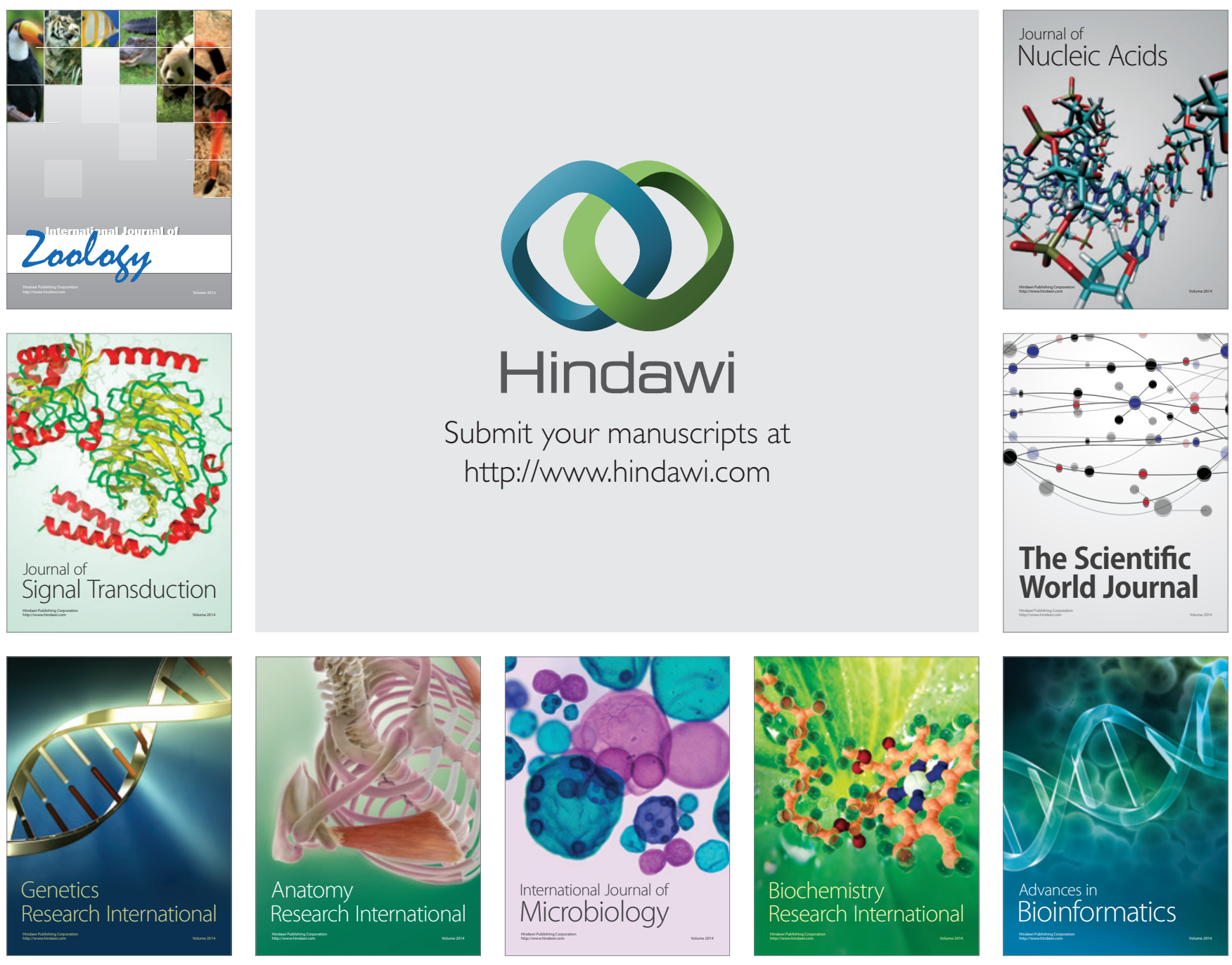

The Scientific World Journal
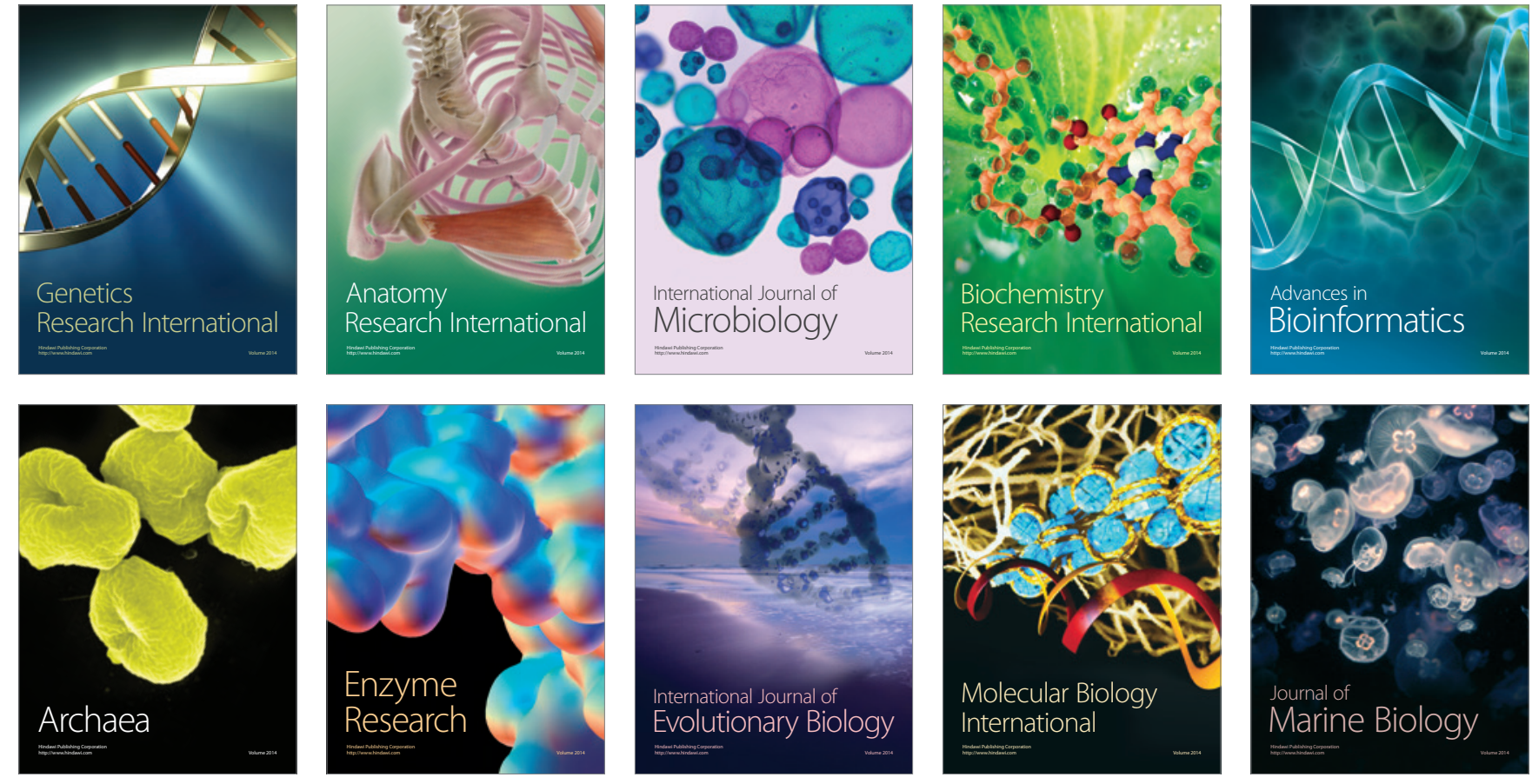